\title{
Head/Neck Squamous Cell Carcinoma: - Prevention Strategy
}

\author{
BM. MANDONG FMCPath * \\ JA. NGBEA $(M B B S)$ * \\ AS. ADOGA** \\ Department of Pathology Jos University Teaching Hospital PMB 2076, Jos * \\ Department of E.N.T Surgery. Jos University Teaching Hospital PMB 2076, Jos ** \\ Correspondence to: BM MANDONG \\ P.O. BOX 887 JOS, PLATEAU STATE, NIGERIA. \\ E-MAIL mafala2004@yahoo.com
}

\begin{abstract}
:
Background:- Head and neck squamous cell carcinoma is the most common histological subtypes of Head and neck tumour. It consist of 4-5\% of all cancer and the fourth leading cause of cancer death in developed and developing nations of America and Africa.
\end{abstract}

Objective:- To describe the epidemiological pattern of Head and Neck squamous Cell Carcinoma (HNSCC) in Northern Nigeria and to review risk factors and prevention strategies.

Methods:- Review of literature published from northern Nigeria from 2000 to 2010. The authors also reviewed strategy for cancer prevention with special emphasis to tobacco cigarette as the major risk factors. Search; head and neck tomours, squamous cell tomours, strategies for prevention.

Key Words:- Squamous cell carcinoma, Tobacco cigarette, Northern Nigeria

\section{Introduction:}

The term "cancer of the head and neck describes a diverse collection of cancers of varying histology arising from varied anatomic sites that make up the upper airo digestive track (UADT). The UADT consists of a complex mucosa covered by squamous epithelium that serves as conduct for food and air that extends from the vermiform surface of the lips to the cervical esophagus.

In common usage this terminology has been applied primarily to those cancers arising from the mucosal surface of the lips, oral cavity, pharynx, larynx and cervical esophagus. Included in this designation however are other important sites such as nose, paranasal, sinuses, salivary gland, thyroid and parathyroid and skin. Some cancers arising in this region are typically excluded from the generic designation of head and neck cancers such as tumours of central nervous system.

Head and neck cancers globally constitution $5-50 \%$ of all cancers when other neck structures such as thyroid, parathyroid, skin, paranasal and nasal sinuses are involved. However, when only the traditional upper airodigestive tract are involved it may constitute $3-5 \%$ of cancers. ${ }^{1-6}$

In Nigeria with low data on cancer incidence, hospital based data from North central Nigeria, Eastern and south western Nigeria show an annual rate of 20-38 cases of head and neck cancers. ${ }^{2-3}$
Some other African countries show a relative high incidence in ages below 40years.

In Jos, North central Nigeria the commonest head and neck cancer occurred in the oral cavity, nasopharynx, larynx and vocal cards. Cancers arising from these sites were squamous cell carcinomas. $^{1-3}$

Head and neck cancer is reported to be the fifth most common cancer worldwide. In America head/neck cancer accounts for $3 \%$ of all new cancer cases and $2 \%$ of all cancer death. In Nigeria and Africa, there is paucity of data as all information being hospital based. ${ }^{7,8,9}$

While the cancer may appear relatively rare in developed nations of America and Europe, It is significantly higher in developing countries like Nigeria with some centers reporting up to $30 \%$ of all cancers. ${ }^{6,7,10}$ Tobacco, alcohol and infections are the primary etiologic agents in Head and neck squamous cell carcinoma. The article is aimed at reviewing the distribution of Head/Neck squamous cell carcinoma globally and strategy for the prevention of tobacco associated HNSC.

Descriptive Epidemiology/Incidence: The five commonest site of HNSCC is mainly from oral cavity, Nasopharynx, Larynx and vocal cord, paranasal sinuses and pharyngeal carcinomas. In Jos North Central Nigeria these are predominantly found in men in the ratio of 4:8: in favour of men. The incidence in men is double for oral-pharyngeal 
cancer and triple in laryngeal cancer. ${ }^{7,8,10}$ The median age at diagnosis for HNSCC is approximately 60 years but the incidence in the young group appears to be increasing. ${ }^{8}$

While in U.S.A. and Britain, the cancer is relatively rare however it accounts for the fifth position in cancer incidence. In, Asia, China and part of Africa HNSCC account for between $17-25 \%$ to causes of all cancers. ${ }^{8,9}$

There is a both regional and intercontinental variation of HNSCC in the World. In America and other developed Nations, laryngeal cancer accounts for one- third of head and neck cancer ${ }^{1,2}$, while southern and Eastern Europe approximately $40 \%$ of all head and neck cancers are laryngeal cancers ${ }^{8}$. In China; Nasopharyngeal cancer accounts for $55 \%$ of all head and neck cancer. However in South Esthern Asia, Nasopharyngeal cancer account for $70 \%$ of all head and cancers and $5 \%$ of all cases of cancers. ${ }^{11,12}$

There is paucity of prevalence and incidence rate of HNSCC in Africa than white however, the overall mortality accounts for $20 \%$ of all cancers ${ }^{9}$. Oral and pharyngeal carcinoma are the commonest in Africa with high mortality ${ }^{12,13}$. The reasons for high mortality may be related to late presentation and low level of community immunity.

\section{Risk Factors:-}

\section{Tobacco:}

Tobacco is the single most important risk factor in the causation of HNSCC. ${ }^{11,13}$ Tobacco initiates a linear dose response carcinogenic effect in which duration is more important than the intensity of exposure'. The major carcinogenic activity of cigarette smoke resides in the particulate (tar) fraction which contains complex mixture of interacting cancer initiators, promoters and cocarcinogens. In the late $1950 \mathrm{~s}$ a landmark case study by Dr. Ernst Wynder established the link between tobacco use and oral cavity cancer ${ }^{11}$. These studies also demonstrated elevated risks for HNSCC deaths in cigar and pipe smoke. Independent case cancers studies for the past 40 years have demonstrated consistently the relationship between tobacco smoking and HNSCC with significant relative odds ratio in 3-12 fold range. Furthermore, a dose response effect is consistently shown in these studies between the duration and dose of smoking with increasing risk of HNSCC and between the time since quitting and the decreasing risk of HNSCC. ${ }^{8}$
While smoking rates are declining in the developed World, smoking rates are rising in developing World, home to four fifth of the world population. In U.S.A and Europe cigarette smoking has been on the decline since 1964 though there is high rate of smoking among school teenagers and increase in the number of cigar users in past few decades. ${ }^{12,13}$ There has been a dramatic increase in the use of smokeless tobacco in young men and has since been implicated in the risk of oral cancers. ${ }^{14}$ Striking difference in variation of HNSCC site and incidence seen among different region, culture and demographic groups, are largely due to different pattern of tobacco and other substance abuse. Smokeless tobacco and other similar products are used greatly in parts of Asia and Africa. ${ }^{13,14}$ In south Central Asia, (betel nut, lime, catchu and areca nut) "Pano" is commonly chewed and is a strong risk factor independent of tobacco used for carcinoma of oral cavity and HNSCC. ${ }^{14}$

\section{Alcohol:-}

Alcohol is an important promoter of carcinogenesis and is reported to contribute $75 \%$ in HNSCC. ${ }^{8}$ furthermore, alcohol appear to have an effect on HNSCC independent of cigarette smoke, but these effects are consistently significant only at highest levels of alcohol consumption. ${ }^{8,11}$ It is believed that ethanol is the principal causative factors and its high level of consumption potentiates the effect of tobacco smoking at every level of its exposure.

\section{Infection Agents:-}

While it has been suggested that various infectious agents play a role in head and neck squamous cell carcinogenesis, only Epstein Barr virus (EBV) and Human papiloma virus (Hpv) can be implicated as etiologic agents in HNSCC based on the current scientific evidence. ${ }^{15}$ EBV appears to be associated with most nasopharyngeal carcinoma, and HPV (mostly type 16, 18) is associated with approximately $50 \%$ of oral laryngeal carcinoma. ${ }^{16}$ Helicobacter has been suggested as a risk factor for laryngeal carcinoma. ${ }^{15}$

THE ROLE OF DIET. Epidemiologic evidence from traditional case study design suggest that diet high in animal fat and low in fruits and vegetable may be risk factor for HNSCC. ${ }^{9}$ It is suggested that vitamin A and beta carotene may be responsible for the protective effects of diets in fruits and vegetables.

Other risk factor is occupational exposure to carcinogens especially workers in leather/textile industries and woodwork factories. Radiation increases the risk of developing HNSCC. 
Prevention Strategy and Early Detection of Tobacco Associated HNSCC

Tobacco smoke is said to be one of the most dangerous substances known to man. ${ }^{18,19}$ The use and consumption of tobacco is almost as old as man.

More than 90 percent of HNSCC is associated with tobacco smoking. ${ }^{20,21}$ The smoking rate is inversely related to the level of education, being more common in young uneducated teenage to low socio-economic status. ${ }^{21}$

The risk assessment of tobacco smoke has been classified as either mainstream or passive (side stream smokers).

Among other constituents, polycyclic Aromatic (PAHS) hydrocarbons and tobacco specific Nitrosamine (TSNAS), nicotine, the second most important abundant constituent of tobacco smoke, it is responsible for the addictive properties of tobacco and reservoir for the services of Tobacco specific Nitrosamine. Nicotine is present both inactive smoker and passive smoker, and is rapidly absorbed by the alveolar of the lungs. Nicotine concentrates in the pulmonary veins as a bolus and circulates throughout the body, subsequently release of dopamine via activation of cholinergic receptors in the brain and modulation of hormones such as epinephrine and cortisol which is believed to lead to Nicotine dependence. The effect of polymorphisms in the dopamine transporter and dopamine receptor genes on smoking initiation and Nicotine dependence remained controversial and a subject of intense investigation. ${ }^{23,24}$

\section{Approach to Prevention and Rehabilitation of Smoke Addiction}

There are two broad approaches to the prevention and treatment strategies.

Prevention programmed for would be tobacco smoker, second treatment plan for cessation of tobacco smoking and rehabilitation.

The most effective way to reduce the rate of mortality attributable to tobacco related cancer is to prevent smoking initiation. Since most smokers begin the use of tobacco under age of 18, tobacco prevention effort must be directed to children and adolescents. ${ }^{20}$ Successful tobacco prevention initiatives depends on an understanding of the specific factors that determine smoking initiative.

Factors that determine the initiating process of tobacco smoking falls into four categories.

- Personality, for example, depression or low self esteem; Family that is having parents who are smokers; peer influence from friends; all these factors increases susceptibility to smoking.

- Phase of experimentation; this is likely if the child or adolescent is exposed to peer encouragement, has poor parental monitoring or parental disapproval exposure to commercial advertisement and may exhibit curiosity about the consequences of tobacco smoking.

- During the phase of experimentation, expectation about the consequences of tobacco use for example relief of anxiety, or depression, peer acceptance developed that shape attitude about continued uses.

- Physiological and addition factors develop and serve to mold casual smoking into regular, sustained use. ${ }^{24}$

\section{Tobacco prevention:-}

This can be approached from two broad bases.

- School based education and counseling programmes

- Community based programmes, the school base programmes were designed to promote awareness of the harmful effects of tobacco use, based on the assumption that if children were aware of the adverse health effect of tobacco smoking, they would abstain.

Some researchers suggest that social influence based prevention programme demonstrate a $50 \%$ reduction in the rates of smoking initiatives up to 6 years following the completion of the programme with the use of booster sessions, Socratic method of instruction, social skill training, individual and group sensitivity and the involvement of parents and siblings, being critical element for programme success. $^{22,23}$

Community with prevention programmes involve mass media education [eg anti smoking billboards, and television commercials] and legislative policy [eg restricting advertising and access, raising taxes, and instituting band], to influence individual behaviours and societal norms concerning adolescent smoking. ${ }^{24}$ Research report has shown that these methods have significantly reduced tobacco smoking rate. ${ }^{25}$ Some data suggest that mass media education can have an independent effect on youth tobacco use. Counter advertising approaches like the "Truth" campaign have been linked with nearly $20 \%$ reduction in smoking among high school students. Qualitative analyses show that counter-advertising message that emphasize tobacco industry manipulation rather than the immediate and long term health consequences of smoking are more effective with 
the youth. In another study it was demonstrated that restricting access to tobacco products to minor and penalties for non-adherent vendors may further reduce tobacco smoking. This study does not prove that it translates into overall reduction in tobacco use. $^{25}$

One study reported that smoking bans at home, in public places, and at school yielded significant reduction in youth tobacco use. ${ }^{20}$ Whereas the effectiveness of smoking bans is influenced by the degree of enforcement, may be converting a cultural norm such that "smoking is no longer socially acceptable may be more effective. ${ }^{22}$ Therefore, there is good reason to believe that counter advertising, restricting tobacco advertising and youth access to tobacco programme may be helpful. However, all these factors may be necessary in the future research for the evaluation and each programme impact assessment on tobacco prevention.

\section{Approach for Smoking Cesation:-}

Despite the growth in the literate on smoking interaction, the prevalence of smoking among adults has not decreased substantially and it has increased among certain adolescent groups. Nevertheless, since the majority of adults and adolescent smokers want to quit, it is critical that effective smoking cessation treatments be developed and disseminated. However, for the past decade emphasis has been on the evaluation of behavioural change and pharmacologic approach to cessation management.

The Agency for Health Care Research and Quality [AHEQ] had carried out a research on various tobacco smoking cessation interventions.

\section{It Comes out with the Following:-}

- Brief tobacco cessation intervention of 3 to 5 minutes can be effective.

- There is a dose response relationship between the intensity and duration of behavioral smoking cessation and effectiveness.

- There are five first line pharmacotherapy that increase quit rate.

- Clinician and health care programme must ensure that all smokers are identified and provided with appropriate treatment.

- Insurance should reimburse patients and physician for the provision of cessation of treatment. Over all, the updated guideline indicates that the most effective smoking treatment involves the combination of tailored behavioral and pharmacologic treatment. ${ }^{24}$ The effects of these treatments are independent and addictive with the combination of the two yielding the highest quit rate.

\section{There are five strategies for cessation programme for tobacco smoking:- \\ - SelfHelp Method \\ - Formal smoking cessation clinics \\ - Physician Based Interventions \\ - Pharmacological Intervention \\ - Harm Reduction.}

\section{Self help method:-}

The most common self help approach to treating nicotine addiction involve the use of self help guides, namely booklets, or pamphlets that describe methods for quitting smoking. ${ }^{20,21}$

Self help method has been viewed as a promising treatment for Nicotine addiction since they are very popular with smokers and can be easily and cheaply disseminated. However, convincing evidence indicated that the quit rate produce by self help guides, can be enhanced when telephone counseling is included as an adjunct.

Formal smoking cessation clinics:- Most cessation programs available to smokers involve some form of behavioural counseling, either individual or group and their effectiveness at producing long term abstinence has been wisely demonstrated. In fact, compared to no treatment the use of behavioural counseling yield cessation rates of $14 \%-17 \%$. Of greater interest is research aimed at identifying the specific types of behavioural counseling that yield the greatest quit rate although such analyses has been hindered by the extreme heterogeneity among studies. ${ }^{25}$ However, three types of behavioural counseling are particularly effective for promoting quitting.

- The provision of problem- solving or skills training, including techniques to avoid relapse and manage stress.

- The provision of intra treatment social support, such as encouragement, understanding and concern.

- The provision of extra treatment, social support from friends and family.

\section{Physician based intervention:-}

With upwards of $80 \%$ of all smokers visiting a physician at least once a year, physician delivered smoking cessation intervention represents a cost effective way to disseminates smoking cessation messages and services to a large number of 
smokers. This approach to treating Nicotine addiction may also be especially, effective since smokers perceive their physician as a valuable credible and reliable source of health information and show a high level of adherence to physician health advance.

\section{Pharmacological intervention:-}

Several studies support the use of five pharmacotherapies as first line treatments for nicotine addiction. These are Nicotine germ, Nicotine patch, Nicotine nasal spray, nicotine inhaler and buprofen hydrochloride. ${ }^{24}$

These have yielded very little success, less than $10 \%$ quit rate

However, it should be noted that the most effective Nicotine addiction therapy are those that combine both behavioural and pharmacological treatment. ${ }^{25}$

\section{Refereeces:}

1. Bhatia Pt. Head and Neck cancer In Plateau State of Nigeria. West Afr. J.Med.1990;9:304-310

2. Nwawolo CC, Ajekigbe AI Oyeneyin JO, et al. Pattern of Head and Neck cancers among Nigerians in Lagos. West Afri. J. Med. 2001;20:111-116

3. Otoh EC, Johnson NW, Mandong BM, Danfillo IS. Primary Head and Neck cancers in Jos Nigeria. A revisit; West Afri. J. med. 2005;24:23

4. Otoh EC, Johnson NW, Danfillo IS, et al Primary Head and neck cancers in North Easthern Nigeria West Afri. J.med. 2004;23:305-313

5. Otoh EC, Hohnson NW, Ajike SO, Mohammed A.Z, et al: Primary Head and Neck cancers in North Wertern Nigeria. West Afri. J.med 2009;28:227-233

6. Amusa YB, Olabanji JK, Ogundipe OV, et al. Pattern of Head and Neck Malignant tumour in a Nigerian Teaching Hospital- a ten year review. West Afri. J.med. 2004; 23:280-285.

7. Nasopharyngeal cancer at the University college Hospital, Ibadan cancer registry: An update. West Afri. J. med. 2004; 23:135-138

8. Carniol Pj. Head and Neck carcinoma in patients under 40 years of age. Ann otol 1982;91:152-155

9. Yu MC. Nasopharyngeal carcinoma epidemiology and dietary factors. In relevance lueman cancer of $\mathrm{N}$ Nitroso componends, tobacco smoke and mycotoxins. Qneill IK, Chen J. Bartch H. (eds). IARC Sci publ. No 105. 1995;P.39-47

10. Otoh EC; Report of the epidemiological survey of head and neck cancers in Nigeria:1972-2002. RCOERH/FMOH Nigeria 2006.
11. Franceschi S, Talamini R, Barra S, et al smoking and drinking in relation to cancers of the oral cavity, pharynx larynx and esophagus in Northern Italy. Cancer Res. 1990; 50:502-507

12. Schantz SP, Yu GP. Head and Neck cancers incidence trends in young Americans, 1973-1997, with a special analysis for tongue cancer. Areh Otolaryngos Head Neck surg 2002;128:268-74

13. Schlechi NF; Franco El, Pintob 3, Kowalski LP. Effect of smoking cessation and tobacco type on the risk of cancer of the upper eoro-digestive tract in Brazil, Epidemiology 1999, 10:412-8

14. Wubb AM. Smokinless tobacco and cancer epidemiologic evidence. CA cancer J. Clin 1988;38:236-43

15. Aygene E. Selcuk A, Celikkanat S, et al. The role helicobacter pylori cell carcinoma of the larynx. 2001; 125:520-521.

16. Mckaig RG, Baric RS, Olshan AF. Human Papilloma virus and head and Neck cancer. Epidemiology and molecular biology. Head Neck 1998;20:250-65

17. Mineta H, Ogino T, Amano HM et al. Human papillomavirus type 16 and 18 detected in head and neck squamous cell carcinoma. Anticancer Res 1998;18:4765-47-68.

18. Engstrem PE, Clapper M, Schnoll RA Orleans CT. Pevention of tobacco related cancers pg. 127-40. In Holland If Frei E, et al. editors. Cancer medicine $5^{\text {th }}$ ed halminton BC Decker 2000.

19. Mullins R. Borland R. Do smokers want to quit? AusT. N.Z J. Public Health 1996;20:426-7

20. Centre for disease central and prevention (2000a) youth risk behavior surveillance united State Morb-mortal WKLY Rep. 2000;49-96

21. Centre for disease central and prevention (2001) cigarette smoking among Adults. United State. Morb mortal WKLY Rep. 50.869-873.

22. Cinciripini PM, McClure JB. Smoking cessation. Recent development in behavior and pharmacological intervention. Oncology 1998;12:249-265.

23. Abrams D. Orleans CT. Niaura RS et al, Integrating individual and Public health perspective for treatment of tobacco dependence under managed care. A combined stepped-care and matching modes. Ann Behav. Med. 1996;18:290-204

24. Lancaster F, Snead LF. Self-help intervention for smoking cessation. Cochrane Database system. Rev. 2000:

25. Kotlyer M, Golding M, Hatsukami DK, Jamerson BD, Effects of non-nicotine pharmacotherapy on smoking behavior. Pharmacotherapy 2001;21:1530-1548. 